\title{
From parallel to serial processing: A computational study of visual search
}

\author{
EYAL COHEN and EYTAN RUPPIN \\ Tel-Aviv University, Tel Aviv, Israel
}

\begin{abstract}
A novel computational model of a preattentive system performing visual search is presented. The model processes displays of lines, reproduced from Wolfe, Friedman-Hill, Stewart, and O'Connell's (1992) and Treisman and Sato's (1990) visual-search experiments. The response times measured in these experiments suggest that some of the displays are searched serially, whereas others are scanned in parallel. Our neural network model operates in two phases. First, the visual displays are compressed via standard methods (principal component analysis), to overcome assumed biological capacity limitations. Second, the compressed representations are further processed to identify a target in the display. The model succeeds in fast detection of targets in experimentally labeled parallel displays, but fails with serial ones. Analysis of the compressed internal representations reveals that compressed parallel displays contain global information that enables instantaneous target detection. However, in representations of serial displays, this global information is obscure, and hence, a target detection system should resort to a serial, attentional scan of local features across the display. Our analysis provides a numerical criterion that is strongly correlated with the experimental response time slopes and enables us to reformulate Duncan and Humphreys's (1989) search surface, using precise quantitative measures. Our findings provide further insight into the important debate concerning the dichotomous versus continuous views of parallel/serial visual search.
\end{abstract}

The visual system of a surviving organism must supply fast answers to burning issues, such as detecting a target in the visual field and characterizing its primary features. An attentional system employing a constant-speed beam of attention (Tsal, 1983) probably cannot perform such tasks fast enough, and a faster, preattentive system is required. Treisman's feature integration theory (FIT) describes such a system (Teisman \& Gelade, 1980). According to FIT, features of separate dimensions (shape, color, orientation) are first coded preattentively in a location map and in separate feature maps, each map representing the values of a particular dimension. Then, in the second stage, attention "glues" the features together, conjoining them into objects at their specified locations.

FIT has been studied with the visual-search paradigm (Treisman \& Gelade, 1980), in which subjects are asked to detect a target within an array of distractors that differ on given physical dimensions. As long as the target is significantly different from the distractors in at least one dimension, the reaction time (RT) is short and shows almost no dependence on the number of distractors (low RT slope). This result suggests that the target is detected preattentively, with all items examined at once, in paral-

We express our gratitude to J. M. Wolfe and an anonymous referee for their helpful comments. We also thank I. Meilijson for his help with the analysis. Correspondence concerning this article should be addressed to E. Cohen, Tel-Aviv University, Department of Psychology, Tel Aviv 69978, Israel (e-mail: eyalc@devil.tau.ac.il).

-Accepted by previous editor. Myron L. Braunstein lel. However, if the target and distractors are similar or the target's specifications are more complex, RT grows considerably as a function of the number of distractors (Duncan \& Humphreys, 1989; Treisman \& Gormican, 1988), suggesting that the display's items are scanned serially in an attentional process.

Throughout this work, we shall deal with the transition from parallel to serial processing. Although FIT originally claimed a parallel/serial dichotomy, subsequent studies have suggested a more gradual transition (Duncan \& Humphreys, 1989). RT slopes are much reduced in conjunction tasks whenever the features of the target are considerably discriminable (Treisman \& Sato, 1990). The picture becomes even more complex owing to the involvement of top-down guidance adding to the bottom-up processes - for example, with the use of categorical information (a steep line target among shallow orientation distractors; Wolfe, Friedman-Hill, Stewart, \& O'Connell, 1992). Another top-down process--namely, object recognition - that might have some influence on the speed of processing has been avoided here by using tasks with displays of lines only, this being the simplest object available and one widely agreed to be processed at the early, parallel stage. Still, one cannot completely avoid topdown processes, as is manifested in our results.

The distinction between parallel and serial processing raises the notion of global versus local scan of visual information: Parallel displays can be processed almost instantaneously, since the preattentional system may efficiently utilize global information embedded in the displays. In serial displays, on the other hand, global in- 
a

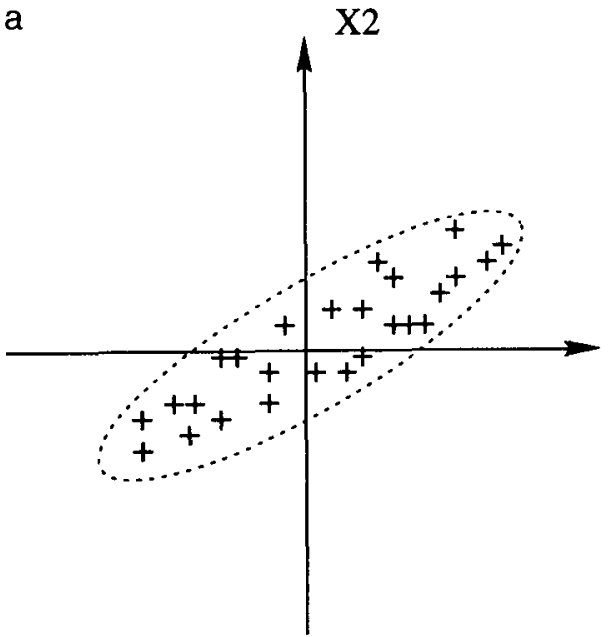

b

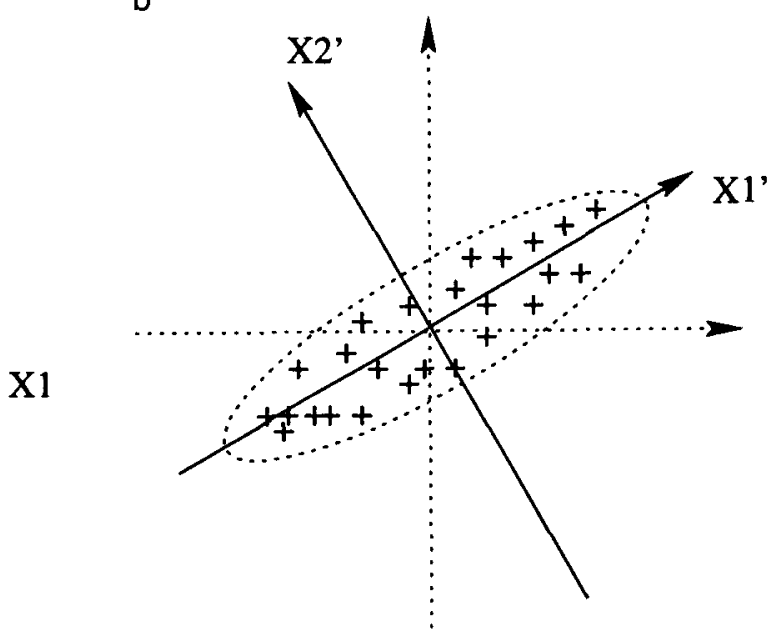

Figure 1. Principal component analysis performed on an ellipse-shaped distribution of data. (a) The original axes system. (b) The new system of axes.

formation may be obscure, and hence, their processing requires serial scanning of localized features. These intuitive notions have served as the cornerstone for the computational study presented in this paper. Our approach is basically motivated by the observation that the visual environment overflows with diverse information, yet the biological information-processing systems analyzing it have limited capacity (Tsotsos, 1990). This apparent mismatch suggests that data compression should be performed at an early stage of perception, retaining only a few essential global features of the visual display. We hypothesize that parallel processing relies on global features that enable fast target detection and study this hypothesis by processing visual displays, using a conventional information-compression method of global natureprincipal component analysis (PCA).

FIT and other related cognitive models of visual search are formulated on the conceptual level and do not offer a detailed description of the processes involved in transforming the visual scene from an ordered array of data points into given values in specified feature maps. This paper presents a novel computational description of such a process, dealing with the transition from parallel to serial processing by employing analyses on visual displays. It also yields a computational interpretation of feature similarity between objects.

In the next section, we provide an overview of the model and the procedures employed throughout the simulations. Our results are described and then discussed, in the perspective of previous models, in the following sections. Finally, a description of the connectionist, neural network realization of target detection is provided in Appendix A. Formal descriptions of the transformation of visual displays into display-vectors and performing PCA are given in Appendices B and C.

\section{THE MODEL}

Our computational model involves two stages of processing. First, an attempt is made to extract global features of the visual displays via PCA. Second, the existence of a target in the display is examined on the basis of this global, compressed information. We first describe the PCA, then describe the visual-search tasks studied, provide a general overview of the model (deferring its formal description to the appendices), and finally, describe the simulation procedures.

\section{Principal Component Analysis and Visual Search}

PCA is essentially a transformation of a data set from its original variables (axes) into new ones. ${ }^{1}$ In the new principal axes system, the information content of the data is reflected more clearly, since the axes are chosen in accordance with the variances of the data. The main principal axis is chosen in the direction of maximal variance of the original data distribution, and the other axes are chosen with decreasing order of variance. For example, the original axes system $\left(x_{1}, x_{2}\right)$ with a tilted ellipseshaped data distribution (Figure 1a) will be transformed via PCA to a new system of axes $\left(x_{1}^{\prime}, x_{2}^{\prime}\right.$; Figure $\left.1 b\right)$. The main principal axis, $x_{1}^{\prime}$, will be aligned with the ellipse's main axis of symmetry, and $x_{2}^{\prime}$ will be aligned in the direction of the ellipse's secondary axis of symmetry. As a by-product, this process yields first-order compression of the data by eliminating redundancies that are present in the original representations of the data.

Each principal axis is provided with an eigenvalue, a number that is equal to the variance of the projections of the data points on that axis. A second-order compression may be obtained by choosing the axes with the largest 
eigenvalues and ignoring the rest, a procedure that minimizes the loss of information, while compressing the original data. For example, the ellipse distribution above requires $2 \mathrm{~N}$ numerical values in order to fully represent the $N$ data items. Alternatively, one can use only the $N$ values of the projections of the data points' on the main principal axis $\left(x_{1}^{\prime}\right)$ and ignore the other $N$ projections (on $\left.x_{2}^{\prime}\right)$. This yields a $50 \%$ compression of the data, but the information loss is much smaller, since most of the information is contained along the main axis.

In order to perform computational procedures on visual displays, one has to transform them into display vectors (see Appendix B). Projections of these vectors on the PCA system of axes are termed internal representations (see Appendix C). These representations are indeed highly compressed: For each 12,800-pixel display vector, only the projections on the first seven axes have been used in the simulations. In fact, for target detection purposes, the first two principal axes' projections are sufficient.

Two questions are now in order. Are global features of the visual displays, like the existence of a target, contained within the first few principal axes? And does the incorporation of PCA into a model of the preattentional system yield results that are compatible with actual psychological experimental data?

\section{The Simulated Tasks}

To demonstrate the generality of the model in support of its plausibility, our computational analysis is performed on visual-search experiments from two different sources: Wolfe et. al. (1992) and Treisman and Sato (1990).

Wolfe et al.'s (1992) Experiments with Line Orientations. Wolfe et al. (1992) conducted experiments of one-feature search, using line orientations. Eight of these experimental tasks were simulated, denoted as WP1, WP2, WP3, WP4, WS1, WS2, WS3, and WS4 (see Figure 2). The RTs, as a function of set size, measured by Wolfe et al. show that type WPi $(i=1-4)$ displays are scanned in a parallel manner (low values of $1.2,1.8,4.8$, and $6.3 \mathrm{msec} / \mathrm{item}$ for the RT slopes), whereas type WS $i$ displays are scanned serially $(17.5,19.7,24.7$, and $20.5 \mathrm{msec} /$ item).

Treisman and Sato's (1990) one-feature and feature conjunction tasks. To test our model for feature integration, we have simulated and analyzed a conjunction task (size with orientation, denoted here as TS1) versus one-feature tasks: size alone (TP1) and orientation alone (TP2). All tasks were taken from Experiment 2 in Treisman and Sato (1990; see Figure 3). The RT slopes measured by Treisman and Sato suggest a serial scan for the conjunction task ( $10.9 \mathrm{msec} / \mathrm{item}$ for the RT slope value) and very fast, parallel scans for the one-feature tasks (low RT slope values of 1.6 and $0.2 \mathrm{msec} /$ item).

We have also generated and simulated another task (XS), using single orientation lines in three sizes-a medium-sized target with a mixture of small- and largesized distractors.

\section{The Input Displays}

Input displays for the simulations were prepared as computerized digital pictures. Displays for Wolfe et al.'s (1992) tasks were produced using 12 matrices of graylevel values of the basic line modules $\left(0,-10^{\circ}, \pm 20^{\circ}\right.$, $\pm 40^{\circ}, \pm 50^{\circ}, \pm 60^{\circ}$, and $\pm 80^{\circ}$ orientations) and randomly assigning these matrices into a $4 \times 4$ array. These matrices were concatenated row-wise into display vectors 12,800 components long (see Appendix B). Three set sizes have been used $-4,8$, and 12 lines. Input displays for Treisman and Sato's (1990) tasks were prepared in a similar procedure: The six line modules $\left(45^{\circ}\right.$ and $135^{\circ}$ orientations in small, medium, and large sizes) were randomly assigned to the $4 \times 4$ array in accordance with the desired task. Three set sizes of 4,9 , and 16 lines have been used. The proportions of lines and display dimensions were kept as close as possible to those used in the original experiments. The number of items in a display equals the number of distractors+target in target displays. In nontarget displays, the target was replaced by another distractor, keeping a constant set size.

\section{Model Overview}

Our model for preattentive processing is composed of two modules connected in sequence (see Figure 4)--a PCA module that transforms and compresses visual displays and a target detection module that detects the existence of a target within a display.

The system operates in the following way: A visual display is presented as an input to the PCA module. This module processes the display and transforms it into a compressed, seven-component internal representation (dark disks in Figure 4). The seven components are then fed into the target detector module that, solely on the basis of this information, decides whether the original display contains a target or not. As we shall show, target detection performance is dependent on the amount of overlap between target and nontarget internal representations.

The system as a whole operates in two phases. Each module first undergoes a training phase until the desired level of performance is achieved on a training set of displays. The training of the PCA module is performed by employing standard mathematical routines to compute the principal axes, using a set of displays of four different tasks in all set sizes. The training phase of the neural network target detector is described in detail in Appen$\operatorname{dix} \mathrm{A}$. Then, in the test phase, the model is "locked," and the composite system is tested on a new, different set of displays from all the simulated tasks.

The crucial issue in the training phase is to choose the correct subset of tasks for the computation of the principal axes (on which all the displays are projected to form the internal representations). One is interested in choosing a relatively small subset of tasks, to demonstrate the systems' ability to generalize - that is, that it can perform a good PCA decomposition even with unfamiliar, new tasks. 
(a) WP1: $1.2 \mathrm{mSec} / \mathrm{ITEM}$

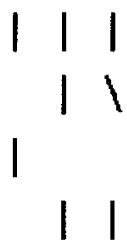

(b) WP2: $1.8 \mathrm{mSec} / \mathrm{ITEM}$

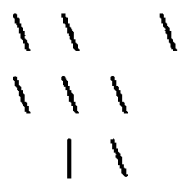

(c) WP3: $4.8 \mathrm{mSec} / \mathrm{ITEM}$

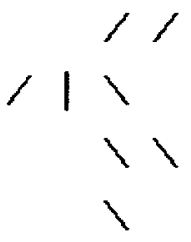

(d) WP4: $6.3 \mathrm{mSec} / \mathrm{ITEM}$

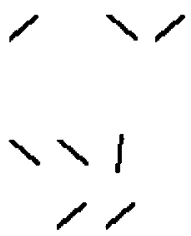

(e) WS1: $17.5 \mathrm{mSec} / \mathrm{ITEM}$

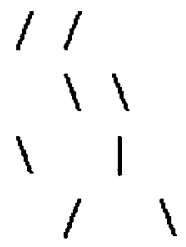

(f) WS2: $19.7 \mathrm{mSec} / \mathrm{ITEM}$

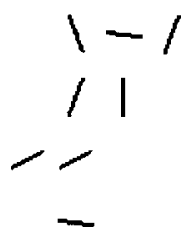

(g) WS3: $24.7 \mathrm{mSec} / \mathrm{ITEM}$

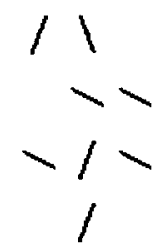

(h) WS4: $20.5 \mathrm{mSec} / \mathrm{ITEM}$

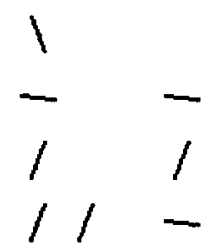

Figure 2. Visual-search displays of line orientation tasks (Wolfe et al., 1992). Note that, in parallel displays, the target "pops out" without being specified. (a) WP1, a $20^{\circ}$ tilted target among vertical distractors (homogeneous background). (b) WP2, a vertical target among $20^{\circ}$ tilted distractors (homogeneous background). (c) WP3, a vertical target among two flanking distractor orientations $\left( \pm 40^{\circ}\right)$. (d) WP4, a steep target $\left(-10^{\circ}\right)$ among two flanking distractor orientations $\left( \pm 50^{\circ}\right)$. (e) WS1, a vertical target among two flanking distractor orientations (at $\pm 20^{\circ}$ ). (f) WS2, a vertical target among heterogeneous background (a mixture of lines with $\pm 20^{\circ}, \pm 40^{\circ}, \pm 60^{\circ}$, or $\pm 80^{\circ}$ orientations). (g) WS3, a $20^{\circ}$ target among $-20^{\circ}$ and $60^{\circ}$ distractors. (h) WS4, a $20^{\circ}$ target among $-20^{\circ}$ and $80^{\circ}$ distractors. For mundane reasons, each line module was $32 \times 25$ pixels in size, yielding $128 \times 100$ pixel displays.

\section{Simulations Procedure}

The simulations were performed in three steps. In the first step, a subset consisting of four of Wolfe et al.'s (1992) tasks (the learning set) was used to produce 2,300 display examples in 24 groups that represent different types of displays: For each task and for each of the three set sizes, target and nontarget groups of displays were prepared. These examples have been used to compute a system of principal axes (i.e., to train the PCA module).

Second, for each of the eight tasks of Wolfe et al. (1992; Figure 2), a new set of target and nontarget display vectors have been prepared and then projected onto the above system of principal axes (see Appendix C). Keeping only the most significant projections on the first seven principal axes as the compressed internal representations of the displays, these projections have served as inputs for the target detector.

Third, using the above inputs, the neural network of the second module was trained to perform target detectionthat is, to identify the existence of a target in a display on the basis of its compressed internal representation.

These three steps have been repeated for different learning sets (i.e., choosing different subsets of tasks to compute the principal axes), looking for the best PCA learning 
(a) TP1: $1.6 \mathrm{mSec} / \mathrm{ITEM}$

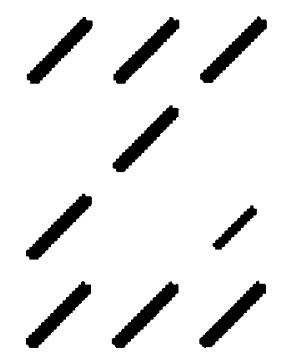

(b) TPE: $0.2 \mathrm{mSec} / \mathrm{ITEM}$

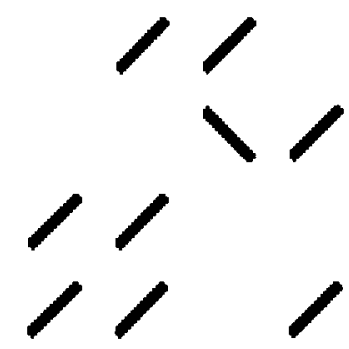

(c) TS1 $10.9 \mathrm{mSec} / \mathrm{TEM}$

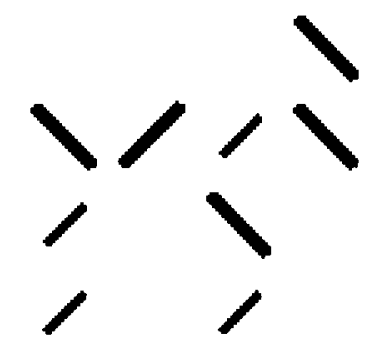

(d) XS: mSec/ITEM

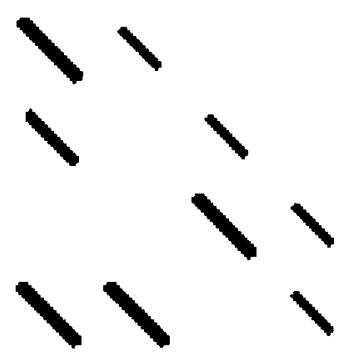

Figure 3. Visual-search displays of one-feature and conjunction tasks. (a) TP1, a small target among large distractors (all tilted $45^{\circ}$ ). (b) TP2, a $45^{\circ}$ target among $135^{\circ}$ distractors (all medium sized). (c) WS1, a conjunction of a $45^{\circ}$ large target with $45^{\circ}$ small and $135^{\circ}$ large distractors. (d) XS, a mediumsized target among small and large distractors (all $135^{\circ}$ ). Each line module is $28 \times 28$ pixels in size, resulting in $112 \times 112$ displays.

basis: the one that yields the strongest correlation between simulated and experimental data-namely, the one for which the target detector succeeds with representations of parallel displays and shows a poor performance with serial ones. This learning basis consists of four vertically symmetric types of displays in all the set sizes: a vertical target among two flanking distractor orientations of $\pm 20^{\circ}$, a vertical target among $\pm 40^{\circ}$ distractor orientations, a vertical target among $\pm 60^{\circ}$ distractor orientations, and a vertical target among $\pm 80^{\circ}$ distractor orientations. Note that only the first two of these types are actual tasks used by Wolfe et al. (1992; WP3 and WS1).

The internal representations that have been computed for the best PCA learning basis have been analyzed extensively, in order to investigate the nature of the transition from parallel to serial processing.

The same procedure and analyses have been employed in Treisman and Sato's (1990) tasks.

All the simulations were performed on Silicon Graphics R- 10000 work stations, with a virtual memory capacity of $5.2 \mathrm{~GB}$, using Matlab5 software.

\section{RESULTS}

\section{Analyses of the Internal Representations}

The results reported hereafter are for the performance of the model with the best PCA learning basis, composed of two of Wolfe et al.'s (1992) tasks (WS1 and WP3; see Figure 2) and two "extra" tasks - one with a vertical target among two flanking distractor orientations at $60^{\circ}$ and another with a vertical target among two flanking distractor orientations at $80^{\circ}$. For Treisman and Sato's (1990) learning basis, all three tasks (TP1, TP2, and TS1) have been used.

Inspection of the values of projections for target and nontarget displays revealed that the second axis was primarily dedicated to target information and hence, was used for the following analysis. ${ }^{2}$

Distributions of the internal representations. For each task and each set size, we have generated two histograms (for target and for nontarget displays) depicting the distribution of the internal representations-that is, the displays' projection values on each of the seven principal axes. Figure 5 presents the projections onto the second principal axis of some of Wolfe's parallel and serial tasks $(a, c)$, and of Treisman's serial conjunction task (b). Ciearly, for parallel tasks, the PCA internal representations of target and nontarget displays form separate groups along that axis (Figure 5a), making the detection of target existence easy. For serial tasks, on the other hand, the two groups grossly overlap (Figure $5 \mathrm{c}$ ), making target detection highly erroneous. For intermediate RT slope values, the two groups are partially overlapping (Figure $5 \mathrm{~b}$ ), and target detection is possible but may require 


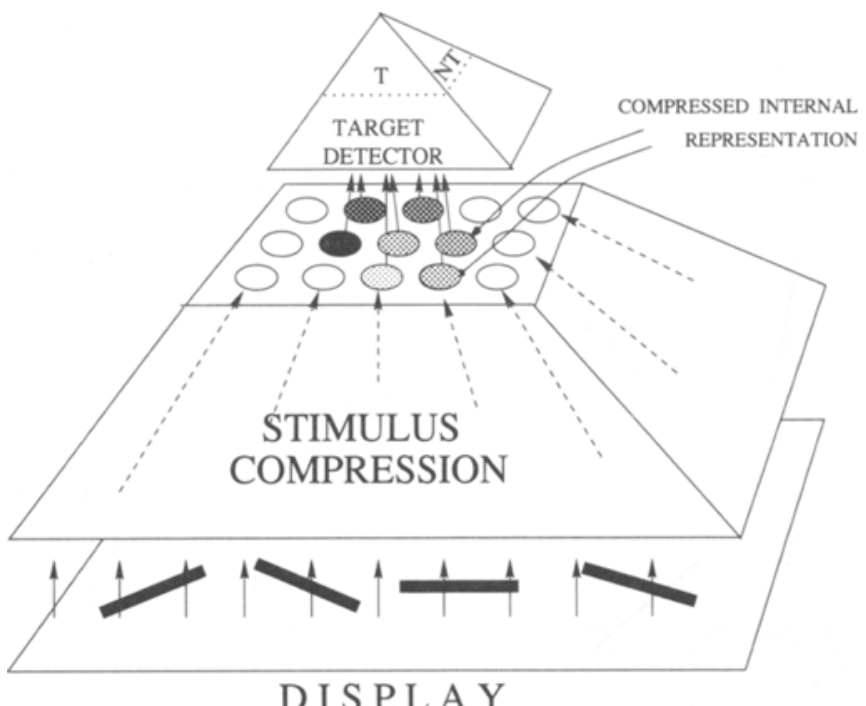

Figure 4. General architecture of the model: the data compression PCA module and the target detector.

longer RTs and may sometimes be erroneous. These histogram forms are typical for all the analyzed tasks.

These differences are further demonstrated in Figure 6 , depicting projections of display vectors on the subspace spanned by the first, second, and third principal axes. Clearly, for parallel tasks (Figure 6a), the PCA internal representations of the target displays (plus signs) are separated from nontarget representations (circles), whereas for serial tasks (Figure 6b), there is no such separation. This description is persistent with all the simulated tasks.

The overlap index. As is evident from the histograms in Figure 5, in parallel tasks, the spreading of the projections' values for target and for nontarget groups of displays is small, as compared with the separation between the two groups. For serial tasks, on the other hand, the spreading of each group is larger, and the two groups of display projections grossly overlap. The degree of over- (a) WP1

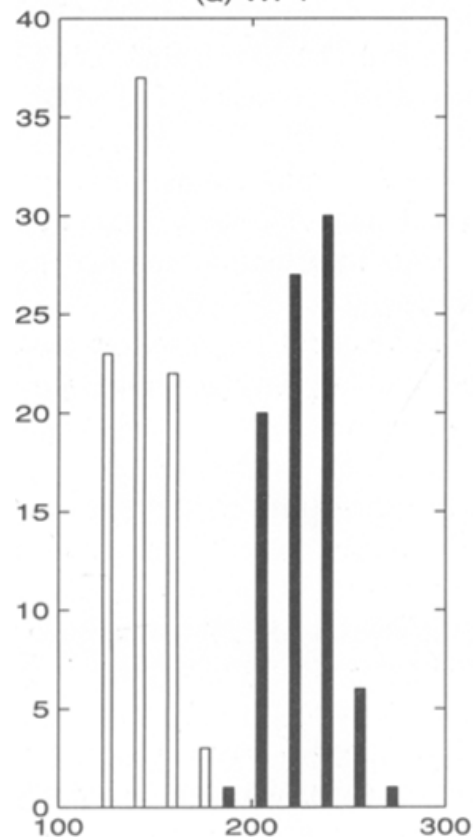

(b) TS1

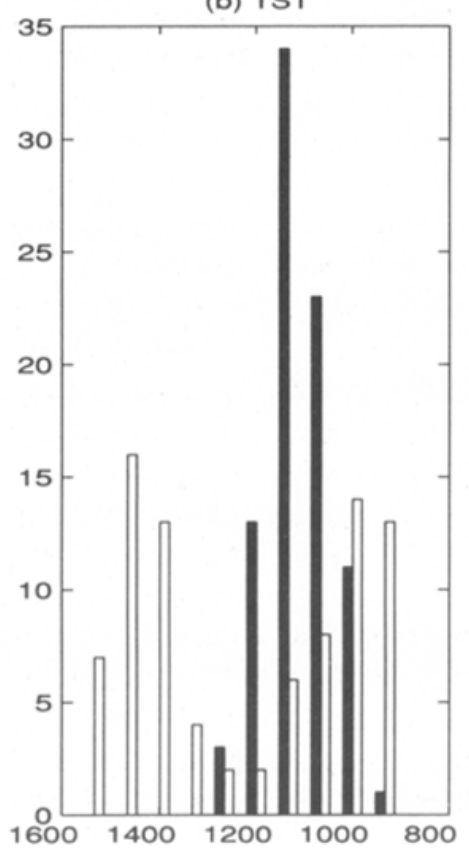

(c) WS4

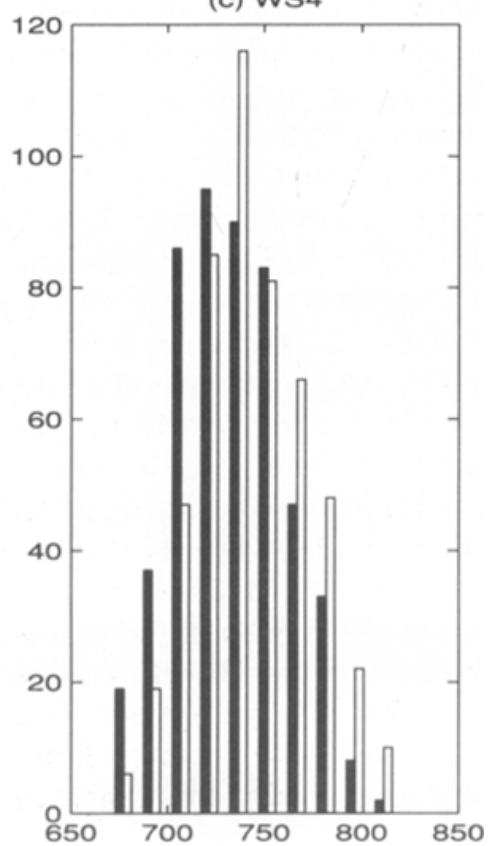

Figure 5. Histograms of projections on the second principal axis of nontarget (white columns) and target display vectors (black columns). Response time (RT) slopes (in milliseconds/item) are (a) 1.2 (parallel), (b) 10.9 (intermediate), and (c) 20.5 (serial). Each group of target or nontarget displays contains 85 examples in (a) and (b) and 500 examples in (c). Set size is eight items. 
(a)WP1

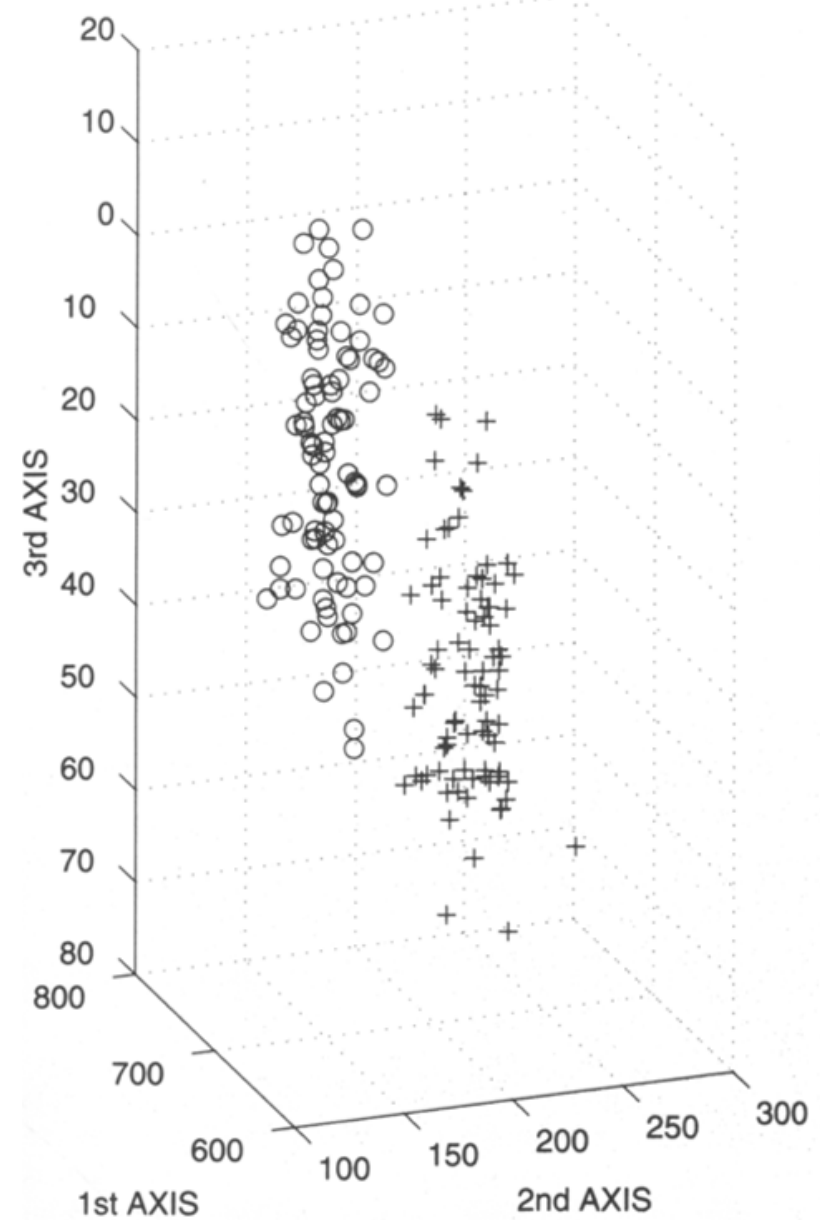

(b)WS3

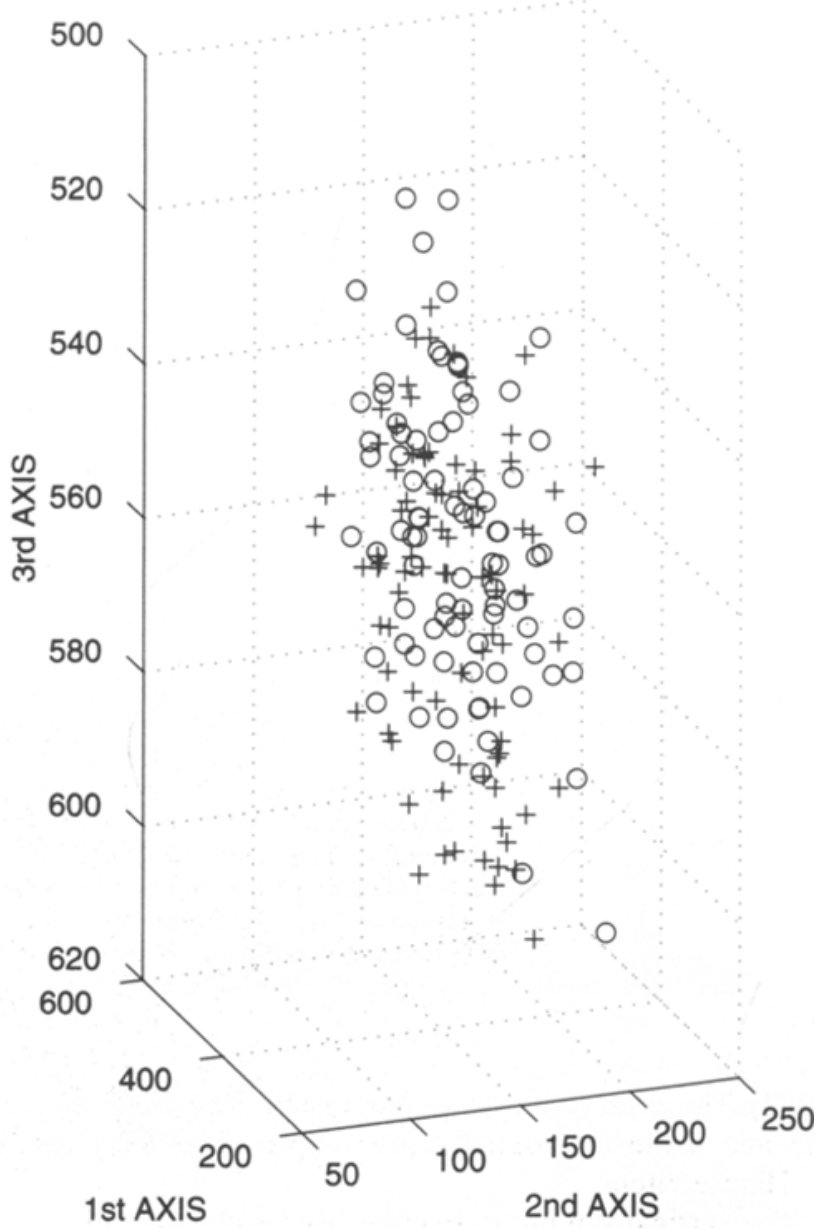

Figure 6. Projections of display vectors on the subspace spanned by the first, second, and third principal axes. Plus signs and circles denote target and nontarget display vectors, respectively, for (a) a parallel task (WP1) and (b) a serial task (WS3). Set size is eight items.

lap between the projections of target and nontarget displays can be stated in quantitative statistical terms, to serve as a parameter distinguishing between parallel and serial displays. To this end, we define the overlap index $Q .^{3}$

To find out whether $Q$ reflects the amount of processing required for successful target detection, we have presented the following hypothesis: The average value of $\mathrm{Q}$ over the three set sizes of each task, being a measure of the mean separability of target and nontarget internal representations, is correlated with the experimental $R T$ slope values. This hypothesis has governed our search for the best learning basis. Namely, we searched for a subset of tasks that forms a PCA system of axes on the basis of which the projections of displays from all the simulated tasks yield $Q$ values that are strongly correlated with the RT slopes.

The strongest $Q$ /RT-slope correlation for Wolfe et al.'s (1992) tasks was obtained by choosing the learning basis set described in the section on Analyses of the Internal
Representations. This choice has an interesting underlying rationale: It includes only tasks with the vertical symmetry characterizing natural scenes. ${ }^{4}$ For Treisman and Sato's (1990) tasks, since none of them is vertically symmetric, all three tasks have been used as the PCA learning basis.

The resulting experimental RT slopes, as a function of the $Q$ index, are displayed in Figure 7. As evident, the $Q$ values are linearly correlated with the RT slope, with a high correlation coefficient of $r_{p}=.79$ (despite the deviation of task WS1). Note that, in general, parallel and serial one-feature tasks form different, compact groups $(Q<$ .05 for parallel tasks and $Q>.7$ for serial tasks). This finding is in accordance with the same separation found in RT slopes (RT slope $<7$ for parallel tasks and RT slope $>17$ for serial tasks). The two-feature conjunction task (TS1), however, has intermediate values on both axes, in agreement with findings from the last few years, indicating that search for a conjunction target is often more efficient than a serial, self-terminating search (Wolfe et al., 


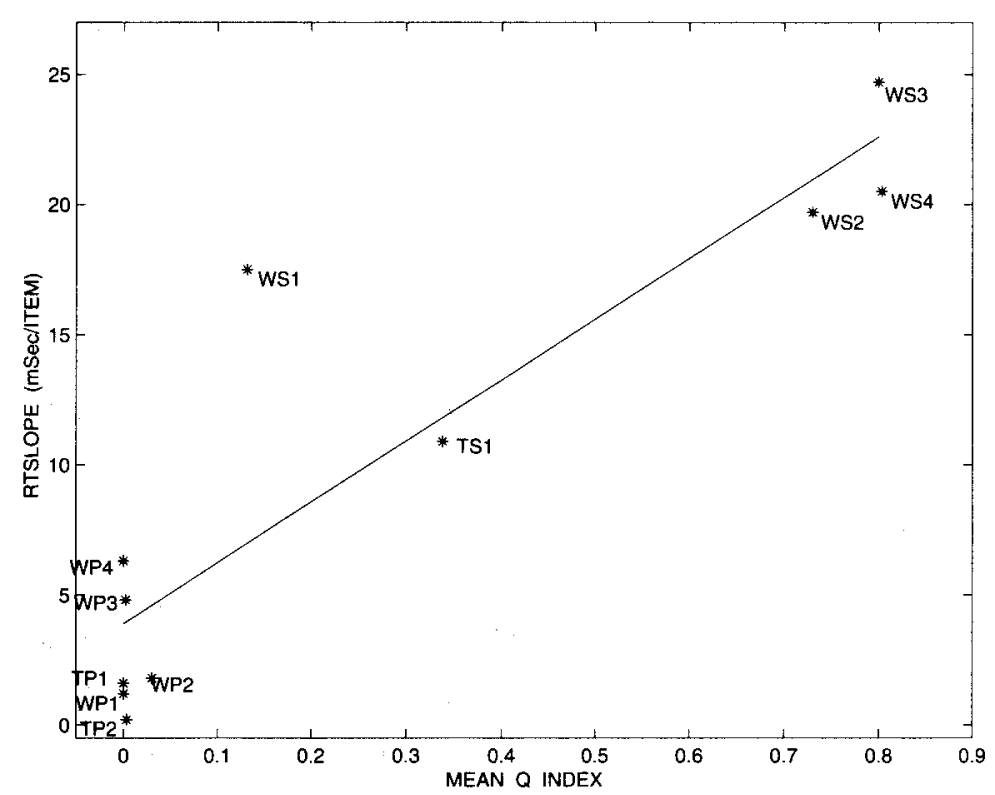

Figure 7. Response time (RT) slopes of Wolfe's (Wolfe, Friedman-Hill, Stewart, \& O'Connell, 1992) experimental data versus the $Q$ index (for the second principal axis). The correlation coefficient between the two variables is $r_{p}=.79$. Each $Q$ value has been computed using 15,000 display examples for each parallel task, and 300,000 examples for each serial task, yielding negligible variances of $Q$ values. The solid line depicts a best-fit curve.

1992). The extra task we have generated (XS) yields $Q=$ .25 , and on the basis of this result, we predict an RT slope of $10 \mathrm{msec} /$ item.

The vertical symmetry that has been embedded in the principal axes is revealed when comparing task WP3 with WS3 and when comparing task WP4 with WS4. In both pairs, the two tasks differ only with respect to the vertical axis of symmetry (see Figure 2) but yield different $Q$ values in accordance with their different RT slopes. Furthermore, in both pairs, the parallel task is the one with a target that is steeper with respect to the vertical axis, as in Wolfe et al. (1992).

Search efficiency. The relation between search efficiency, on the one hand, and the distinction between parallel and serial processing, on the other hand, has been investigated by Duncan and Humphreys (1989), who claim that there is no such distinction and that search efficiency varies continuously across tasks. The determining factors, according to Duncan and Humphreys, are the similarities between the target and the nontargets $(T-N$ similarity) and the similarities between the nontargets themselves (N-N similarity). Displays with homogeneous background (high $\mathrm{N}-\mathrm{N}$ similarity) and a target that is different from the distractors (low T-N similarity) will exhibit parallel, low RT slopes, and vice versa. This claim was illustrated by them with a qualitative search surface description, as is shown in Figure 8a. On the basis of our results, we can now examine this claim quantita- tively. Evidently, for parallel task displays, the withingroups variance is small, as compared with the betweengroups variance (Figure 5a), and vice versa for serial tasks (Figure 5c). ${ }^{5}$ Therefore, we have redrawn Duncan and Humphreys's search surface, using actual numerical data of RT slopes from Wolfe et al.'s (1992) experiments, replacing the $\mathrm{N}-\mathrm{N}$ similarity axis with the within-group standard deviations of the PCA-compressed representations and N-T similarity with the between-group standard deviations (Figure 8b). Treisman and Sato's (1990) tasks have not been included in the surface, since they contain lines of different proportions that lead to different scales in standard deviation values. The resulting surface is partially similar to Duncan and Humphreys's surface. However, according to our analysis, there is a strong monotonic dependency of search efficiency on the within-groups standard deviations but a U-shaped dependency on between-groups standard deviations. This result suggests that $\mathrm{T}-\mathrm{N}$ and $\mathrm{N}-\mathrm{N}$ similarities are not the best choice for determining search efficiency and that another parameter (e.g., the $Q$ index) should be used.

\section{Target Detection}

Taking the same compressed internal representations that have been used for the analysis, we have trained the neural target detector. Using the seven leading principal axes, we find that, for parallel tasks (WP1, WP2, WP3, WP4, TP1, and TP2, with $Q$ values $<.05$, the network 
a

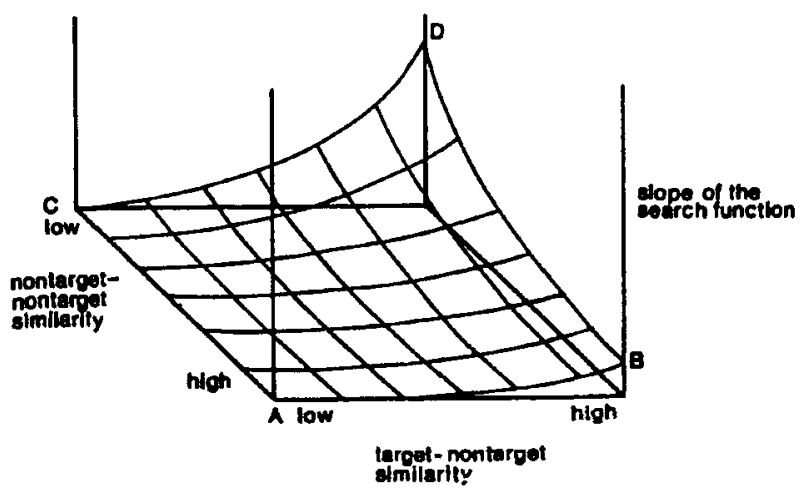

b

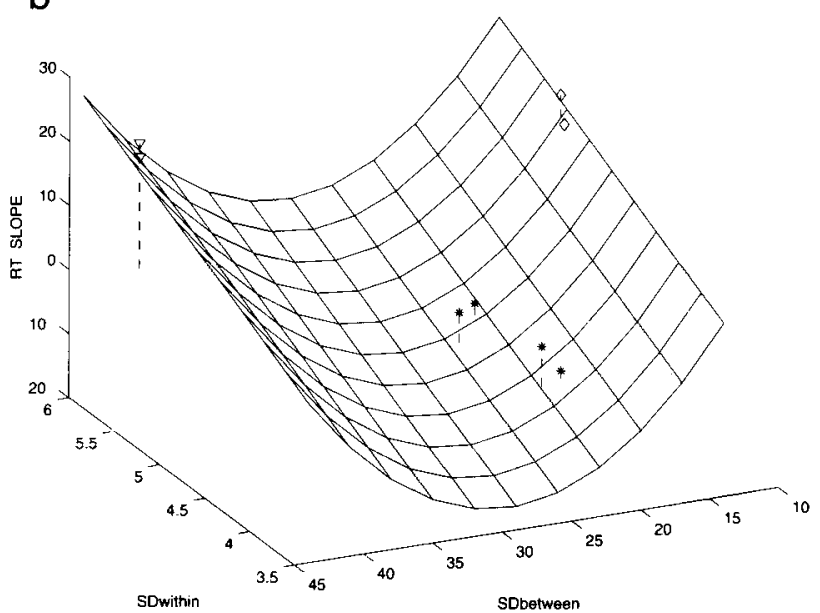

Figure 8. Response time (RT) slopes versus (a) input similarities (the search surface, reprinted from Duncan \& Humphreys, 1989) and (b) standard deviations of the PCA variance analysis. Asterisks denote tasks WP1-4, triangles denote WP1-2, and diamonds denote WP3-4.

detects the existence of targets with a $100 \%$ level of correctness. For serial tasks (WS1, WS2, WS3, WS4, TS1, and $\mathrm{XS} ; Q>.1$ ), the network performs erroneous target detection. The smallest error rate that can be achieved by the network in the training process of each task is the measure of the target detector's performance. Six of these values are presented in Figure 9, compared with six of Wolfe et al.'s (1992) error rates (for tasks WP1, WP2, WP3, WS1, WS2, and WS3; Treisman and Sato, 1990, report only, in general, an error level of less than $6 \%$, without specifying values for individual tasks). The different error rates are depicted in Figure 9 as a function of the RT slopes, and in both cases, we find a positive monotonic relation between RT slopes and the error rates (in fact, Treisman and Sato's [1990] report of a $6 \%$ error fits well too but cannot be explicitly depicted). However, the performance of the neural target detector, although starting at the lowest possible error values with parallel tasks, deteriorates to unacceptable rates with serial tasks, as compared with human performance. This finding strongly suggests that a model, based solely on utilizing global information, can operate well only with parallel tasks and that serial tasks require the operation of additional systems.

Finally, Wolfe et al.'s (1992) error rate plots reveal no correspondence between the set size and the error rate within the same task. Similar behavior is observed here with the error rate values of the neural target detector.

\section{DISCUSSION}

In this work, we have presented a computational model of preattentive visual search. The model was applied to the visual-search experiments performed by Wolfe et al. (1992) and Treisman and Sato (1990). Our main finding is that, when global compression is applied to visual-search displays, the amount of overlap between tar- get and nontarget compressed representations is strongly correlated with the experimental RT slopes. This, however, requires a descriptive space of principal axes with an embedded vertical symmetry. In this space, the $Q$ index leads to a dichotomous view of visual-search processing with a possible third group of feature conjunction tasks, allowing one to distinguish between parallel and serial displays as a function of the global information they contain.

On the basis of these findings, we conclude that fast parallel detection of a target among distractors may be successfully performed, utilizing compressed global information only. However, target detection in serial displays requires more information that is not available globally or may be obscure (noisy). The additional attentional systems required for the utilization of the localized information succeed in maintaining a low, constant error rate with serial displays but are evidently time consuming. In this view, conjunction task displays contain partially obscured information that requires additional, local processing. Note that it is different from another view, which argues that conjunction searches produce intermediate slopes because they have imperfect preattentive information to guide the deployment of attention.

Vertical symmetry, a fundamental characteristic of natural scenes, plays a central role in visual-search tasks. This is demonstrated in Wolfe's work (Wolfe et al., 1992): Two tasks, WP3 and WS3, which differ only in a rotation of $20^{\circ}$ about the vertical axis, yield completely different slopes: parallel for the vertically symmetric WP3 and highly serial for WS4. Indeed, the best fit of our model with experimental data (Figure 7) was found using a PCA learning basis (i.e., computing the principal axes), with only vertically symmetric displays (see the section on Simulation Procedure). This may also be the source of the difference between our search surface and Duncan and 


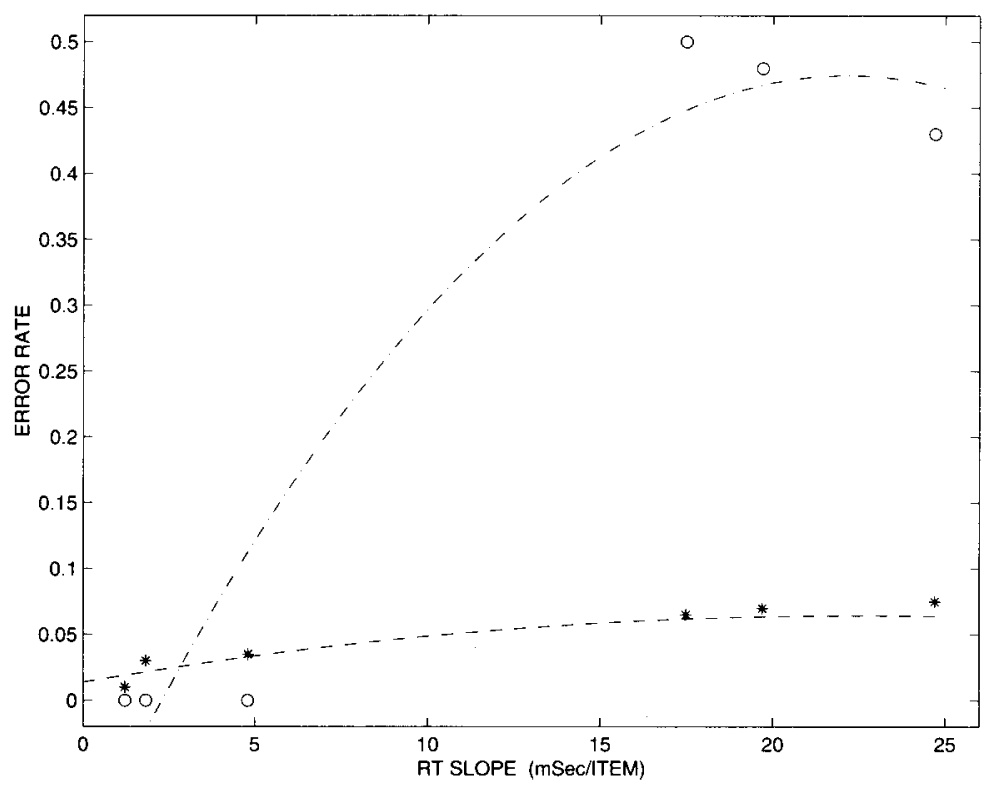

Figure 9. Error rates of the model's target detector (circles) and experimental findings (asterisks) as a function of response time (RT) slope. Dashed and dashed-dot lines are best-fit curves. Error rates are averaged over all set sizes.

Humphreys's (1989): From the point of view of T-N and $\mathrm{N}-\mathrm{N}$ similarities alone, WP3 is identical to WS3, since these similarities do not account for vertical asymmetry. However, on the basis of the present model, it is not possible to determine whether this symmetry is employed as a bottom-up component of visual processing or as topdown guidance.

The model demonstrates generalization ability. The principal axes for Wolfe et al.'s (1992) tasks were computed with a learning basis that contained only two of his parallel tasks. Still, the system performs perfectly on all four of his simulated parallel tasks and with some error rate on his serial tasks.

There are a few algorithms for processing and compressing visual data. We have chosen PCA, its being a standard procedure for efficient representation of global information in displays, since it is known to be a natural manifestation of the Hebbian learning rule in neural networks (Baldi \& Hornik, 1989) and its possible role in perception has already been investigated (Cottrell, 1990; Hancock, Baddeley, \& Smith, 1992).

Our results serve to testify how a computational approach may be complementary to other ways of modeling search performance. First, by incorporating a mathematically rigorous procedure of data compression, one is able to come up with the definite conclusion that serial tasks do not contain enough global information for performing target detection and, hence, other systems employing additional information are required. Second, like other computational models, our model has an immediate neural network realization (see Appendix A) that may serve as a bridge (albeit much simplified) between the cognitive and the biological levels of description.

In summary, this paper utilizes a computational model to show that the distinction between parallel and serial processing may originate from the earliest stages of visual processing. The data we have used come from two different experimental groups (Wolfe et al., 1992, and Treisman \& Sato, 1990) using a variety of experimental tasks and aimed toward different cognitive models. Our model is quantitatively compatible with both groups' data and, to some extent, with the ideas of a third party, Duncan and Humphreys (1989). As is shown here, parallel displays contain global information, in their PCA representations, that enables fast, preattentive target detection. Serial displays do not, thus requiring further processing.

\section{REFERENCES}

Ackley, D. H., Hinton, G. E., \& Sejnowsky, T. J. (1985). A learning algorithm for Boltzmann machines. Cognitive Science, 9, 147-169.

BALDI, P., \& HorNiK, K. (1989). Neural networks and principal components analysis: Learning from examples without local minima. Neural Networks, 2, 53-58.

Cottrell, G. W. (1990). Extracting features from faces using compression networks: Face, identity, emotion and gender recognition using holons. Proceedings of the 1990 Connectionist Models Summer School, 328-337.

Duncan, J., \& Humphreys, G. (1989). Visual search and stimulus similarity. Psychological Review, 96, 433-458.

Hall, E. L. (1979). Computer image processing and recognition. New York: Academic Press.

Hancock, P. J. B., Baddeley, R. J., \& Smith, L. S. (1992). The principal components of natural images. Network, 3, 61-70.

Rumelhart, D. E., Hinton, G. E., \& Williams, R. J. (1986). Learn- 
ing representations by back propagation errors. Nature, 323, 533536.

SANGER, T. D. (1989). Optimal unsupervised learning in a single-layer linear feed-forward neural network. Neural Network, 2, 459-473.

Treisman, A., \& Gelade, G. (1980). A feature integration theory of attention. Cognitive Psychology, 12, 97-136.

Treisman, A., \& Gormican, S. (1988). Feature analysis in early vision: Evidence from search asymmetries. Psychological Review, 95, 15-48.

Treisman, A., \& Sato, S. (1990). Conjunction search revisited. Journal of Experimental Psychology Human Perception \& Performance, 16, 459-478.

TSAL, Y. (1983). Movement of attention across the visual field. Journal of Experimental Psychology. Human Perception \& Performance, 9 , 523-530.

Tsotsos, J. K. (1990). Analyzing vision at the complexity level. Behavioral \& Brain Sciences, 13, 423-469

Wolfe, J. M., Friedman-Hill, S. R., Stewart, M. I., \& O'Connell, K. M. (1992). The role of categorization in visual search for orientation. Journal of Experimental Psychology. Human Perception \& Performance, 18, 34-49.

\section{NOTES}

1. PCA is most commonly used as a factor analysis in the processing of experimental data. However, in this paper, we are using it as an algorithm that compresses stimulus (visual) data.
2. In general, each principal axis contains information of different global features, which may mask the information concerning the existence of a target.

3. Given two distributions (normalized to unity) with some degree of overlap, the joint integral $I$ over all the values of the distributed variable can serve as an overlap measure. For two separated distributions, $I=2$ (the sum of two normalized distributions). For two distributions that completely overlap, $I=1$ (the area of one distribution), and for partially overlapping distributions $1<\mathrm{I}<2$, since the overlapped area is counted only once. In our analysis, we have computed $I$ numerically, using 500-bin histograms, and have redefined the overlap index as: $Q=$ $2-1$. In this case, $0 \leq Q \leq 1$, with $Q=0$ for best separatıon (no overlap) and $Q=1$ for maximal overlap between target and nontarget groups of projections.

4. Despite being isotropic in nature, PCA can reflect symmetries embedded in the visual display - with respect to the vertical line of symmetry (e.g., a vertical target among two flanking distractor orientations of $\pm 40^{\circ}$ ), as well as with respect to an arbitrary axis of symmetry (e.g., a $+20^{\circ}$ target among $-20^{\circ}$ and $60^{\circ}$ distractors). A system of principal axes that exhibits vertical symmetry can be obtained by using only displays of such symmetry in computing the principal axes.

5 . The within-groups variance is a measure of the background homogeneity (the similarity between the nontargets: $\mathrm{N} \cdot \mathrm{N}$ similarity) in a group of displays, whereas between-group variance ( $\mathrm{T}-\mathrm{N}$ similarity) measures the difference between a group of target displays and a group of nontarget displays.

\section{APPENDIX A \\ A Connectionist Realization}

Our model can be described, in neural network terms, as a parallel distribution processing system. The model is composed of two neural network modules connected in sequence (see Figure A1): a PCA module that transforms the visual data into a compressed set of projections along the main principal axes and a decision-making module. The system is presented with line orientation displays transformed into display vectors, as will be described in Appendix B.

\section{The Principal Component Analysis Module}

PCA can be performed by a neural network proposed by Sanger (1989) or by an auto-encoder network (Ackley, Hinton, \& Sejnowsky, 1985; Baldi \& Hornik, 1989). For computational space/time reasons, we have not used neural networks to model PCA throughout the simulations but, rather, have used mathematical routines. The ability of these networks to perform PCA is well established (see also Hancock et al., 1992) and is not at the focus of our work.

\section{The Target Detection Module}

Target detection in our system is performed by using the most significant projections on the first seven principal axes as inputs to the target detection module. The target detection task is performed by a feed-forward (FF) three-layered network, trained via a standard back-propagation algorithm (Rumelhart, Hinton, \& Willaams, 1986). The input layer of the FF network ("TD input"-layer in Figure A1) is composed of the 7 output neurons of the PCA module. Each of these neurons is connected to 12 neurons in the hidden layer, which in turn are connected to a single decision output neuron. Each neuron sums up the activity of all the neurons in the preceding layer. For the hidden layer we get:

$$
h_{j}=\sum_{k=1}^{7} w_{j k} s_{k},
$$

where $w_{j k}$ is the synaptic weight of the connections between neuron $j$ and the $k$ th neuron in the preceding layer and $s_{k}$ is the activity of the $k$ th neuron. This sum of activities is processed with a hyperbolic tangent activation function [with asymptotic values of $( \pm 1)]$ :

$$
s_{j}=\tan h\left(h_{j}\right)
$$

yieldng the $j$ th neuron's activation, which is then transmitted to the next layer.

The network is trained in a supervised-learning manner in which the correct response to each display $(+1$ for a target and -1 for a nontarget) is given during the training phase. For each display, the output error $\delta_{\text {output }}$ 


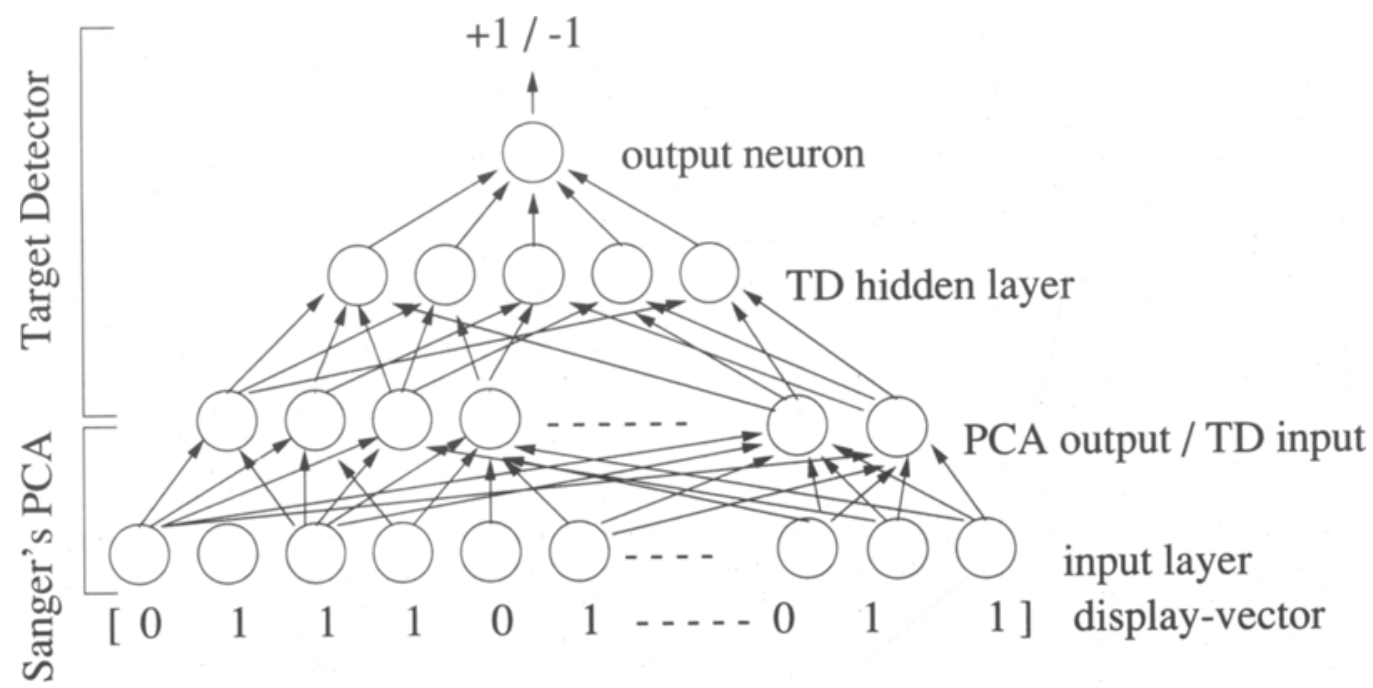

Figure A1. A sketch of the neural model's architecture. For clarity of exposition, not all connections are depicted.

(equal to the difference between the correct output and the actual output) is computed, and the connections' values are updated, using the back-propagation learning rule:

$$
\Delta w_{i j}=\eta \times \sum_{\text {all display vectors }} \delta_{\text {output }} \times V_{\text {input }},
$$

where $w_{i j}$ is the amount of change in the $i j$ connection, $\eta$ is the learning rate (a value of .01 has been used), $\delta_{\text {output }}$ is the output error of a layer, and $V_{\text {input }}$ is the input vector produced by the activation of the preceding layer. This training process is repeated until the error is reduced to a desired level (or the error approaches a nonzero asymptotic value, as with serial tasks). From this time on, the network may be used to detect targets in a new set of visual displays: For each internal representation of a parallel display, it yields an output of +1 or -1 , depending on whether there is a target in the display or not.

(a)

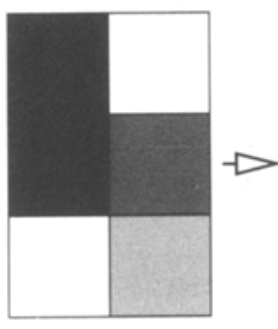

(c)

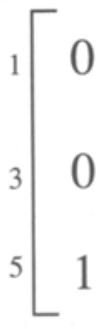

(b)

(d)
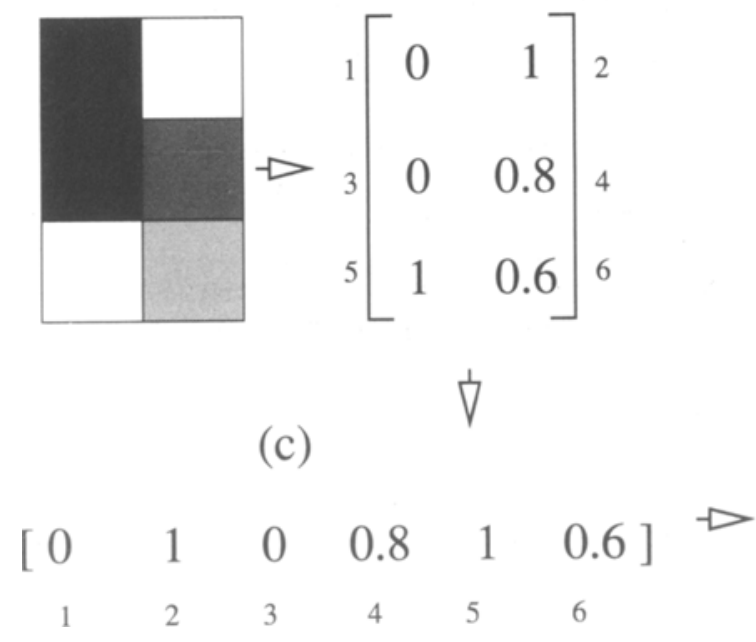

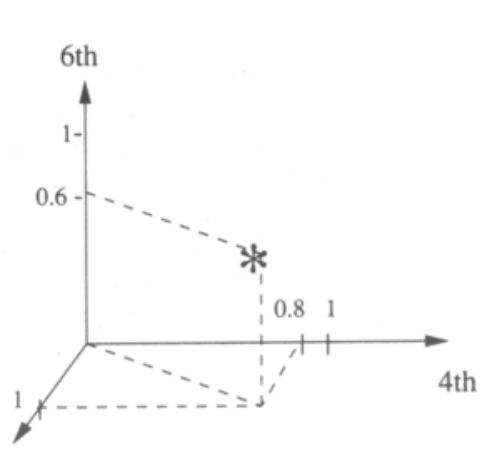

5 th

Figure A2. A vector representation of a $3 \times 2$ visual display. (a) A gray-level display. (b) A matrix of graylevel values. (c) Concatenating the matrix into a row vector $\Rightarrow$ the display vector; note the order of the elements (small digits). (d) Graphical description of the 4, 5, and 6 components of the display vector in the subspace spanned by the 4-, 5-, and 6-pixel axes. The vector is represented by the asterisk; its projections along the axes (dashed lines) are equal to the gray-level values of the corresponding pixels in the original display. 


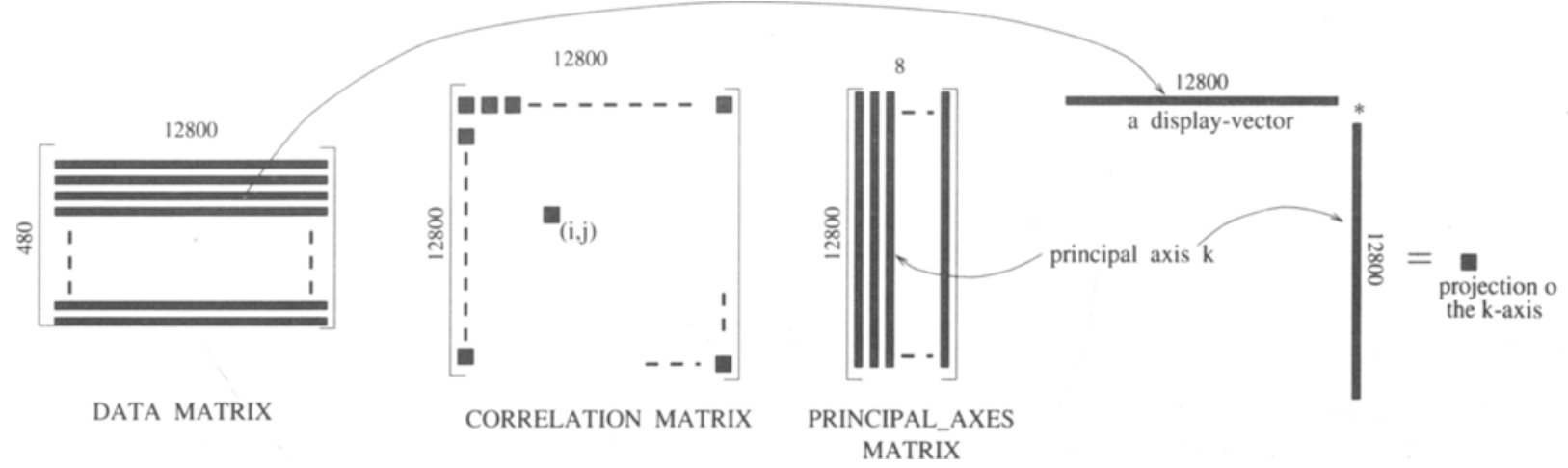

Figure A3. Principal component analysis performed on data of 480 display vectors, each 12,800 long, compressed to eight component vectors of global, internal representations.

\section{APPENDIX B \\ Vector Representations of Visual Displays}

In order to perform computational procedures on visual displays, they have to be transformed into vectors. Throughout this work, we have used displays of black lines on a white background, with $N=128 \times 100=$ 12,800 or $N=112 \times 112=12,544$ pixels in each, which were transformed into 12,800 (or 12,544) components, single row vectors (Figures A2a-c). We have termed these vectors display vectors, and all our computations use them as faithful representatives of the visual displays. These vectors may also be described in a space of $12,800(12,544)$ dimensions, as in Figure A2d.

\section{APPENDIX C \\ Principal Component Analysis Computations}

In order to perform PCA on a set of 480 displays, $128 \times 100$ pixels each (numbers here are chosen for convenience of presentation), one has to create a data matrix with 480 rows (Figure A3); each row is a display vector 12,800 elements long, as was described in Appendix B. Then, a 12,800 $\times 12,800$ correlation matrix is computed where each $(i, j)$ element of this matrix is equal to the correlation between the values of the $i$ th pixels of the 480 display vectors and the values of the $j$ th pixels.

Solving the eigenvalues equation (a standard algebraic routine; see Hall, 1979, for a detailed exposition) for the correlation matrix, one finds the principal axes matrix accompanied by a set of variances along each principal axis - the eigenvalues. The columns of the latter matrix are orthogonal vectors that represent the principal axes in the following way: Multiplying a display vector with the $i$ th column yields the projection value of this display on the direction of the $i$ th principal axis. Each of the original display vectors can now be projected onto these new axes, and the new component values constitute the representation of the visual display in the PCA space. This representation is referred to as the PCA internal representation of the display, and it is highly compressed, since we are using no more than seven principal components out of a 12,800-pixel display! 